\title{
Synchronous Primary Brain Tumors in the Pediatric Age Group. Report of Two Cases and Review of the Literature
}

\author{
Omar Chamdine ${ }^{1}$, Marwah Abdulkader ${ }^{1}$, Wissam Issawi ${ }^{2}$, Shaymah Al-Umran $^{1}$, Youssef \\ Housaawi $^{1}$, and Mahmoud Taha ${ }^{1}$ \\ ${ }^{1}$ King Fahad Specialist Hospital Dammam \\ ${ }^{2}$ Imam Abdulrahman Bin Faisal University King Fahd Hospital of the University
}

May 7, 2020

\begin{abstract}
Synchronous brain tumors are rare in children. We present 2 unique cases of synchronous brain tumors and we elaborate on their presentation, diagnosis, approach to management, and outcome. The first case describes a unique and previously unreported combination of a supratentorial anaplastic ependymoma and a cerebellar low-grade glioma.

Synchronous Primary Brain Tumors in the Pediatric Age Group. Report of Two Cases and Review of the Literature

Omar Chamdine ${ }^{1}$, Marwah Abdulkader ${ }^{2}$, Wisam Al-Issawi, MD ${ }^{3,4}$, Shaymaa Al-Umran ${ }^{4}$, Youssef Housaawi ${ }^{5}$, Mahmoud Taha ${ }^{3}$

${ }^{1}$ Department of Pediatric Hematology Oncology and Stem Cell Transplantation, King Fahad Specialist Hospital, Dammam, Saudi Arabia

${ }^{2}$ Department of Pathology, King Fahad Specialist Hospital, Dammam, Saudi Arabia

${ }^{3}$ Department of Neurosurgery, King Fahad Specialist Hospital, Dammam, Saudi Arabia

${ }^{4}$ Department of Neurosurgery Imam Abdulrahman bin Faisal University

${ }^{5}$ Department of Genetics, King Fahad Specialist Hospital, Dammam, Saudi Arabia

AbstractSynchronous brain tumors are rare in children. We present 2 unique cases of synchronous brain tumors and we elaborate on their presentation, diagnosis, approach to management, and outcome. The first case describes a unique and previously unreported combination of a supratentorial anaplastic ependymoma and a cerebellar low-grade glioma.
\end{abstract}

Key words: synchronous, metachronous, brain, tumor, ependymoma, low grade glioma

\section{Key Clinical Message:}

Approach to children with synchronous brain tumors should be individualized and tailored to each patient. It should not be taken for granted that multiple tumors are of the same origin. Treatment significantly varies depending on the histopathology.

\section{Introduction}

Synchronous and metachronous brain tumors (BT) are rare in children and present diagnostic and therapeutic challenges. Besides, malignant transformation (MT) in children is not a frequent occurrence ${ }^{1}$ and can be 
missed if a high index of suspicion is not present. In this report, we present 2 unique cases of synchronous $\mathrm{BT}$ and we elaborate on their presentation, diagnosis, approach to management, and outcome. The first case describes a unique and previously unreported combination of a supratentorial (ST) anaplastic ependymoma (EPN) and a cerebellar low-grade glioma (LGG) in a child. The second case describes the MT in one of two tumors LGGs present in the same patient while the other tumor remained low grade. The first patient remains alive at the time of reporting the case while the second patient succumbed to his disease.

\section{Case Reports}

Case \#1

A 9-year-old girl with no significant family history presented with 2 weeks history of persistent headaches, vomiting and abdominal pain. Physical examination at presentation did not show any neurologic deficits nor any stigmata of genetic predisposition. Radiological studies, including magnetic resonance imaging (MRI) of the brain, revealed two space-occupying lesions. The first lesion was located in left fronto-temporal region and displayed high grade features. The second lesion was located in the right cerebellar hemisphere and demonstrated lower grade features than the former lesion (figure 1). MRI of the spine and CSF analysis did not show evidence of metastatic disease. The patient underwent gross total resection of the left parietal tumor and subtotal resection of the right cerebellar tumor. Histopathological examination of the ST tumor showed a highly cellular glial neoplasm with prominent pseudo vascular rosettes and ependymal like cytological features. The neoplastic cells were rather small, containing pleomorphic nuclei, many mitotic figures with apoptotic bodies identified. Following immune-histochemical studies, the tumor cells were immunoreactive with GFAP with absence of Olig2 expression. Ki67 (proliferative index) was very high (80\%). There were foci of microvascular proliferation as well as tumor necrosis. Molecular analysis was performed on paraffin embedded tissue and the chromosomal microarray analysis identified multiple genomic alterations in the tumor, including gain of chromosomes 8,13 , and 14, copy neutral loss of heterozygosity (cnLOH) from 9p24.3 to 9p13.3 with focal homozygous deletion of 9p21.3 (including CDKN2A and CDKN2B), cnLOH of chromosome 12 and chromothrypsis of chromosome 11. Next generation sequencing (NGS) identified C11orf95-RELA (exon3:exon2) gene fusion. Final diagnosis was "Anaplastic EPN (WHO Grade III), RELA fusion-positive". Microscopic examination of the cerebellar tumor demonstrated a different histological pattern. The tumor was localized and far less cellular, and the neoplastic cells were spindle in shape with oval bland nuclei. No definite Rosenthal fibers or eosinophilic granular bodies were observed. There was no significant increase in mitoses, and no necrosis or microvascular proliferation was present. Immunohistochemically, the cells were positive with GFAP and Olig 2; Ki67 ( proliferative index) was low (2-3\%). BRAF KIA1549 fusion was not detected. The final diagnosis of a pilocytic astrocytoma (PA), BRAF fusion negative was attained. Next generation sequencing (OncoRisk expanded) panel for 89 genes, including CNVs analysis, was performed to investigate any underlying germline mutation but did not detect any pathogenic variants. Following the surgery, the patient suffered from transient weakness in her right arm and leg which resolved within 2 weeks. She received focal radiation (RT) therapy to the supratentorial tumor at a total cumulative dose of 59.4 Grays in 33 fractions. Upon follow up, three and six and months post radiation therapy, there was complete resolution of the first high grade lesion without evidence of disease recurrence. The second tumor in the posterior fossa showed stable residual disease without significant signs of progression. Later on, at nine months follow up, a new dural based lesion in the left temporal area was detected with radiologic characteristics similar to the initial supratentorial tumor. Tumor resection was not feasible due to marked tumor vascularity and risk of bleeding. Biopsy of the dural based lesion showed similar histological and immunohistochemical findings to that seen in the first high grade supratentorial lesion. The patient received treatment with 5 drug metronomic therapy (bevacizumab, celecoxib, thalidomide, alternating oral etoposide and cyclophosphamide). Tumor response to therapy was remarkable, and gross total resection was feasible after 4 months of uninterrupted therapy. The patient was planned to receive craniospinal radiation therapy following tumor resection; however, she developed severe ventriculitis and remains hospitalized for treatment of the infection. She is currently alive at 19 months from initial diagnosis and remains disease free.

Case \#2 
A of 12-year old boy presented with right sided focal seizures. His family history was negative for inherited cancers and did not elicit any clinical or radiological stigmata of any genetic predisposition syndrome. The initial MRI of the brain showed two intra axial tumors in different locations: one in the frontal lobe and another one in the cerebellum. They both exhibited similar radiologic features of low grade lesions (figure 2 ). The family refused any surgical intervention, and he was managed symptomatically with antiepileptic medications and close observation with serial brain MRIs. The condition remained clinically and radiologically stable until five years later when he developed new onset persistent headaches and vomiting. MRI of the brain at that time showed significant increase in the size of the left frontal lobe mass with development of a cystic component and restriction of water diffusion suggestive of MT. The cerebellar lesion did not show significant changes. He underwent subtotal resection of the of the frontal lobe tumor with concurrent biopsy of the cerebellar tumor. Postoperatively, he underwent focal radiotherapy RT to the left frontal lobe lesion at a total cumulative dose of 59.4 Gy with concurrent chemotherapy. Six months later, the left frontal lobe tumor progressed again and he underwent a debulking surgery. He passed away three months after the second surgery. Genetic testing to detect underlying germline mutations was not performed. Histological examination from the first surgical intervention reveals a high grade glioneurnal tumor with calcifications. Immunohistochemical staining showed a mixture of neurons stained with synaptophysin and NSE, while the glial component displayed astrocytic cytological features. There were numerous mitotic figures but no microvascular proliferation or necrosis were present. The diagnosis of an anaplastic ganglioglioma (WHO grade III), negative for $1 \mathrm{p} 36$ and 19q deletions. The second lesion in the cerebellar showed all the classical histopathological features of a pilocytic astrocytoma (WHO grade I), negative for BRAF fusion and BRAF V600E mutation.

Tissue from the second surgery revealed a high grade glioneuronal tumor with hyper cellularity and large areas of necrosis. The neoplastic cells had a more epithelioid cell morphology, with numerous giant cells; mitotic figures were easily identified, reaching 9 per 10 high power fields. Proliferative index Ki-67 was $60 \%$ and P53 stain was diffusely and strongly positive in $60 \%$ of the nuclei. The final diagnosis was glioblastoma (GBM), a giant cell type (WHO grade IV).

\section{Discussion}

The vast majority of cases of synchronous and metachronous brain tumors occurred in adults. There are many reported cases in the literature as early as the $19^{\text {th }}$ century ${ }^{2}$. Histopathologically, the vast majority are constituted of meningiomas and astrocytomas and are more common in patients with known underlying genetic syndromes or after radiation therapy for previous brain tumors ${ }^{3}$. We performed a comprehensive literature review and identified a total of twelve pediatric cases with synchronous/ metachronous brain tumors (table 1 ).

The origin of gliomas remains a matter of investigation. Evidence from mouse models of glioma and human clinical data have provided some clues about proposed cell types in initiating oncogenic mutations that drive gliomagenesis. Some suggested neural stem cells, progenitor cells or differentiated progeny as the cell of origin of different glioma subtypes. The cell of origin is thought to be of neuroglial in lineage and differs from the "brain tumor stem cells" which initiates tumor formation and helps tumor survival and virulence. ${ }^{4}$ The link between these cell types is still not discovered. In our patients the presence of synchronous/ metachronous brain tumors may support this former theory of a common stem cell of origin of gliomas. These stem cells may migrate to different brain locations and thrive in a different microenvironment leading to a specific type of glioma ${ }^{4}$. Cohnheim suggested that embryonic residual cells could evolve into different primary brain lesions ${ }^{5}$.

In this report, we describe two rare cases of children with multiple brain tumors exhibiting different histologic subtypes and occurring simultaneously in the same patient. To the best of our knowledge, case\#1 is the first report of a child with both, a ST anaplastic EPN and a cerebellar PA. One study reported a case of an 8-month-old girl suffering from a frontal lobe ependymoma in association with a cerebellopontine angle meningioma without any genetic predisposition ${ }^{3}$. Upon review of the adult literature, two cases of concurrent meningiomas and EPN were detected ${ }^{6,7}$. 
While MT is frequent in adults (accounting for 50-90\% of high grade glioma cases) ${ }^{8,9}$ it is rare in children. The long-term risk of MT in children is estimated to be less than 10\% (2.9-10\%). ${ }^{1,10}$ Although our second patient did not have histologic confirmation at diagnosis, the clinical and radiologic pictures highly suggested that the original tumor in the left frontal lobe was a LGG that later underwent MT.

Genetic predisposition syndromes tend to be linked to the histogenesis of different intra axial and extra-axial tumors, hence increases the risk of simultaneous neoplasms ${ }^{11}$. Previous RT exposure also predisposes to the occurrence of different primary tumors within the same individual ${ }^{12,13}$, but is otherwise rare ${ }^{3,14}$. Five out of the 12 reported pediatric cases (41.6\%) developed the second tumor 6 to 21 years following RT to the first tumor. RT was focal in 2 cases, craniospinal in 2 cases, and whole brain in 1 case ${ }^{3,5,15-20}$. Case\#1 did not have a genetic predisposition syndrome and both cases were not exposed to either radiation of chemotherapy at the time of diagnosis.

Outcome of relapsed anaplastic EPN is unfavorable. Available treatment options include surgical resection and/ or RT (focal or craniospinal). Chemotherapy is usually ineffective ${ }^{21}$. We elected to treat our patient with metronomic therapy based on a published experience. ${ }^{22}$ Up to date, the tumor growth of this tumor is well controlled. Therapy resulted in a significant decrease in the tumor vascularity, thus facilitating gross total resection without major morbidity. Although the risk of recurrence is high, the patient remains alive and has regained her baseline quality of life.

Conclusion

Histopathologic analysis and molecular interrogation of each lesion in synchronous and or metachronous brain tumors is highly recommended. This will enable the oncologists to offer individualized and tailored therapy and avoid under or over treatment. A high index of suspicion should be present for genetic predisposition in such cases.

\section{Author Contribution:}

1. Conception and design: Omar Chamdine, Mahmoud Taha, Marwah AbdulKader

2. Acquisition of data: Omar Chamdine, Mahmoud Taha, Marwah Abdulkader, Shayma Al-Umran

3. Drafting of manuscript: Omar Chamdine, Marwah Abdulkader, Mahmoud Taha

4. Critical revision: Omar Chamdine, Mahmoud Taha, Marwah Abdulkader, Wisaam Al-Issawi

\section{References}

1. Broniscer A, Baker SJ, West AN, et al. Clinical and molecular characteristics of malignant transformation of low-grade glioma in children. Journal of Clinical Oncology . 2007;25(6):682-689. doi:10.1200/JCO.2006.06.8213

2. Langdon FW. Multiple tumours of the brain; fibrocystoma of pons and cerebellum and multiple fibro-psammomata of dura, pia-arachnoid and cortex cerebri. Brain . 1895;18(4):551-560. doi:10.1093/brain/18.4.551

3. Zenke K, Sakaki S, Nakagawa K. Association of Meningioma and Ependymoma. Neurologia MedicoChirurgica. 1992;32(4):220-4. doi:10.2176/nmc.32.220

4. Modrek AS, Bayin NS, Placantonakis DG. Brain stem cells as the cell of origin in glioma. World journal of stem cells . 2014;6(1):43-52. doi:10.4252/wjsc.v6.i1.43

5. Jea A, Coscarella E, Chintagumpala M, et al. Medulloblastoma and juvenile pilocytic astrocytoma presenting as synchronous primary brain tumors in a child: Case report. Journal of Neurosurgery: Pediatrics . 2010;5(2):149-154. doi:10.3171/2009.9.PEDS09211

6. Molnár P, Hegedüs K. Adjacent astrocytoma and ependymoma: Dependent mixed neoplasia or unusual collision tumors? Surgical Neurology . 1984;22(5):455-460. doi:10.1016/0090-3019(84)90302-1 
7. Rodriguez HA, Berthrong M. Multiple Primary Intracranial Tumors in von Recklinghausen's Neurofibromatosis. Archives of Neurology . 1966;14(5):467-475. doi:10.1001/archneur.1966.00470110011002

8. Vertosick FTJ, Selker RG, Arena VC. Survival of patients with well-differentiated astrocytomas diagnosed in the era of computed tomography. Neurosurgery . 1991;28(4):496-501. doi:10.1097/00006123-19910400000002

9. McCormack BM, Miller DC, Budzilovich GN, Voorhees GJ, Ransohoff J. Treatment and survival of low-grade astrocytoma in adults-1977-1988.Neurosurgery . 1992;31(4):636-642; discussion 642. doi:10.1227/00006123-199210000-00004

10. Mistry M, Zhukova N, Merico D, et al. BRAF mutation and CDKN2A deletion define a clinically distinct subgroup of childhood secondary high-grade glioma. Journal of Clinical Oncology . 2015;33(9):1015-1022. doi:10.1200/JCO.2014.58.3922

11. Vijapura C, Saad Aldin E, Capizzano AA, Policeni B, Sato Y, Moritani T. Genetic Syndromes Associated with Central Nervous System Tumors.Radiographics : a review publication of the Radiological Society of North America, Inc . 2017;37(1):258-280. doi:10.1148/rg.2017160057

12. Little MP, de Vathaire F, Shamsaldin A, et al. Risks of brain tumour following treatment for cancer in childhood: modification by genetic factors, radiotherapy and chemotherapy. International journal of cancer . 1998;78(3):269-275. doi:10.1002/(SICI)1097-0215(19981029)78:3<269::AID-IJC1>3.0.CO;2-T

13. Taylor AJ, Little MP, Winter DL, et al. Population-based risks of CNS tumors in survivors of childhood cancer: the British Childhood Cancer Survivor Study. Journal of clinical oncology : official journal of the American Society of Clinical Oncology . 2010;28(36):5287-5293. doi:10.1200/JCO.2009.27.0090

14. Rieske P, Zakrzewska M, Biernat W, Bartkowiak J, Zimmermann A, Liberski PP. Atypical molecular background of glioblastoma and meningioma developed in a patient with Li-Fraumeni syndrome. Journal of Neuro-Oncology . 2005;71(1):27-30. doi:10.1007/s11060-004-9181-3

15. Smith MDR, Hardman LJM, Earle KM. Contiguous glioblastoma multiforme and fibrosarcoma with extracranial metastasis. Cancer . 1969;24(2):270-276. doi:10.1002/1097-0142(196908)24:2<270::AIDCNCR2820240210>3.0.CO;2-5

16. Tanaka J, Garcia JH, Netsky MG, Williams JP. Late appearance of meningioma at the site of partially removed oligodendroglioma: case report. Journal of Neurosurgery . 1975;43(1):80-85. doi:10.3171/jns.1975.43.1.0080

17. Robinson RG. A second brain tumor and irradiation. Journal of Neurology, Neurosurgery, and Psychiatry.1978:1005-1012. doi:10.1136/jnnp.41.11.1005

18. Pearl GS, Mirra SS, Miles ML. Glioblastoma Multiforme Occuring 13 Years After Treatment of a Medulloblastoma. Neurosurgery.1980;6(5):546-51. doi:10.1227/00006123-198005000-00009

19. Kanoke A, Kanamori M, Kumabe T, Saito R, Watanabe M, Tominaga T. Metachronous, multicentric glioma of pilocytic astrocytoma with oligodendroglioma-like component and oligodendroglioma through distinct genetic aberrations: Case report. Journal of Neurosurgery . 2013;118(4):854-858. doi:10.3171/2012.9.JNS112353

20. Butti G, Giordana MT, Paoletti P, Schiffer D. Multiple primary intracranial tumors of different cell types: Association of anaplastic astrocytoma and acoustic neurinoma - with review of the literature.Surgical Neurology . 1982;18(5):336-342. doi:10.1016/0090-3019(82)90144-6

21. Messahel B, Ashley S, Saran F, et al. Relapsed intracranial ependymoma in children in the UK: patterns of relapse, survival and therapeutic outcome. European journal of cancer (Oxford, England : 1990) . 2009;45(10):1815-1823. doi:10.1016/j.ejca.2009.03.018 
22. Robison NJ, Campigotto F, Chi SN, et al. A phase II trial of a multi-agent oral antiangiogenic (metronomic) regimen in children with recurrent or progressive cancer. Pediatric blood \& cancer . 2014;61(4):636642. doi:10.1002/pbc.24794

\section{Figures}

Figure 1. Axial FLAIR images of the brain showing a supratentorial tumor in the left fronto-temporal area with significant amount of surrounding edema (left) and a right cerebellar lesion with no surrounding edema (right)

Figure 2. Figure 2a Coronal FLAIR images showing right cerebellar (left) and left supratentorial (right) lesions, both showing low grade features. Figure $2 \mathrm{~b}$ showing a coronal T2 image with features of a high grade tumor (MT) of the right supratentorial lesion (right), and an axial FLAIR image showing unchanged right cerebellar lesion (left)

\section{Tables}

Table 1. Summary of reported pediatric cases with multiple brain tumors 


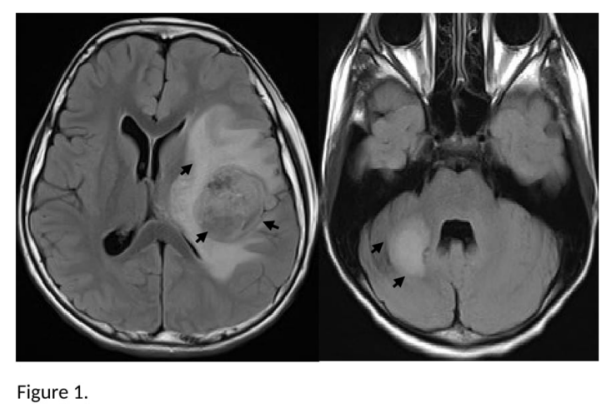




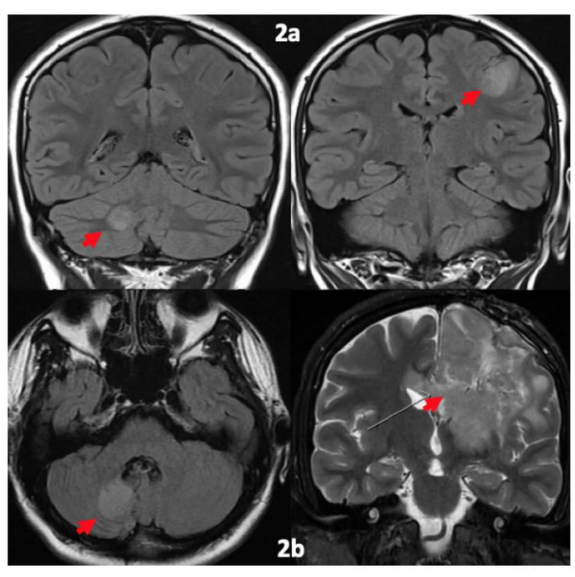

Figure 2. 